ENCYCLOPEDDIE Encyclopédie berbère

BERBERE

$26 \mid 2004$

26 | Judaïsme - Kabylie

\title{
Kabylie : La Kabylie antique
}

\section{J.-P. Laporte}

\section{OpenEdition}

Journals

Édition électronique

URL : http://journals.openedition.org/encyclopedieberbere/1400

DOI : 10.4000/encyclopedieberbere. 1400

ISSN : 2262-7197

\section{Éditeur}

Peeters Publishers

\section{Édition imprimée}

Date de publication : 1 mai 2004

Pagination : 4000-4015

ISBN : 2-7449-0452-X

ISSN : 1015-7344

\section{Référence électronique}

J.-P. Laporte, « Kabylie : La Kabylie antique », Encyclopédie berbère [En ligne], 26 | 2004, document K06, mis en ligne le 01 juin 2011, consulté le 14 décembre 2020. URL : http://journals.openedition.org/ encyclopedieberbere/1400 ; DOI : https://doi.org/10.4000/encyclopedieberbere.1400

Ce document a été généré automatiquement le 14 décembre 2020.

(c) Tous droits réservés 


\title{
Kabylie : La Kabylie antique
}

\author{
J.-P. Laporte
}

1 Les territoires situés entre l'Oued el Kebir (antique Ampsaga*) à l'Est et le bassin de l'Oued Isser (antique Serbétès*) à l'Ouest présentaient dès l'Antiquité des caractères humains particuliers liés sans doute à leur nature montagneuse. Ils se sont ouverts aux différentes cultures, punique, romaine et chrétienne sans renoncer à leur appartenance au monde libyque et sans perdre des identités régionales bien marquées.

2 L'exploration archéologique de la Kabylie du Djurdjura, qui a suivi de peu la conquête sanglante de 1857, a été précoce et approfondie (Laporte 1998) ; elle a été effectuée par des officiers français cultivés et attentifs à la vie, la langue et l'histoire de la région. C'est ainsi que, dès 1868, le capitaine Christian de Vigneral (1868) donnait un inventaire archéologique consistant pour l'ensemble du massif jusqu'à Bougie. Certes les militaires avaient tendance à voir partout des ouvrages militaires, forts, redoutes, etc., dont la plupart se sont révélés être en fait des établissements agricoles, mais ils ont signalé et décrit des ruines isolées dont beaucoup ont disparu depuis. Les civils qui leur ont succédé après 1871, notamment Camille Viré (1898), ont remarqué pour leur part nombre de traces de mise en valeur agricole. Vers 1910, Saïd Boulifa a découvert d'importants vestiges, notamment plusieurs stèles libyques figurées ( $C f$. Laporte 1998, p. 707). Mais l'effort fut interrompu par la guerre de 1914-18 et la région presque oubliée des archéologues. Ce n'est qu'après la seconde guerre mondiale que l'on note des fouilles à Tigzirt (par L. Déroche, E. Frézouls, M. Euzennat, S. Lancel), et, après 1962, un renouveau des prospections par le Père G. Poyto, J.-C. Musso, le Père J. Martin et J.-P. Laporte.

3 L'exploration archéologique de la Kabylie des Babors, dont l'histoire reste à écrire, a en revanche été plus tardive et, à part quelques brillantes descriptions militaires, a été essentiellement l'œuvre de civils rayonnant autour des deux grandes villes, Bougie et Sétif, et exposant leurs découvertes dans le Recueil de la Société Archéologique de Constantine.

4 Malgré tous ces travaux exposant leurs découvertes, les périodes antérieures à la présence punique, échappent encore largement à l'analyse. Tout au plus peut-on dire que la région se trouvait à l'extrémité occidentale du Maghreb oriental, celui des 
haouanet* (à l'exception d'une nécropole isolée à Tipaza, le plus occidental a été signalé à Bougie), et de la zone des dolmens littoraux, qui se raréfient au delà jusqu'à la frontière marocaine (Camps 1995, p. 24-25).

\section{La période libyco-punique}

5 Des négociants phéniciens puis puniques ont fréquenté depuis longtemps la côte. Ils avaient des contacts au moins commerciaux avec la population libyque. La première trace historique apparaît au v viècle avant J.-C. dans le "Périple de Scylax », rédigé vers 415 avant J.-C, avec la citation du toponyme Sida, forme ancienne de Saldae (Bejaia*). Tous les noms des sites côtiers sont puniques (ou pour certains libycopuniques), ce qui montre clairement la précocité de l'influence carthaginoise. Les rares noms connus pour l'intérieur sont exclusivement libyques, notamment celui des trois grands fleuves: la Nasavath (Soummam), l'Addima (Sebaou), l'Usar ou Serbétès (Isser). La Sava (oued Bou-Sellam) était peut-être considérée comme le cours supérieur de la Soummam.

6 À Dellys, une petite monnaie d'or de la Carthage punique des années 320-300 avant J.-C. constitue le plus ancien vestige datable de toute la région. Les villes de la côte étaient fortement punicisées. Igilgili a livré une nécropole de type punique, avec fosses et caveaux creusés dans le roc (Gsell 1911, f. VII, $n^{\circ}$ 77). Mais, non loin de là, la vie libyque continuait à son rythme. La région côtière de la Kabylie du Djurdjura a livré plusieurs monuments mégalithiques d'un type unique dans le reste du Maghreb: de longues allées couvertes signalées à Ibahrissen*, la pointe Ksila, Aït Raouna* et peut-être à Takdempt, près de Dellys. Aït Raouna en offre les exemples les mieux connus. À la fin $\mathrm{du} \mathrm{IV}^{\mathrm{e}}$ et pendant le $\mathrm{III}^{\mathrm{e}}$ siècle avant $\mathrm{J}$.-C, à $10 \mathrm{~km}$ à peine de l'escale punique de Rusazus (Azeffoun), la population libyque enterrait dans ces sépultures collectives les restes de ses défunts, décharnés et/ou incinérés. Leurs ossements étaient enfouis avec des poteries ou fragments de poteries qui pouvaient contenir des offrandes alimentaires. Le bon millier de tessons découverts par J.-C. Musso dans les allées couvertes d'Aït Raouna se composait d'environ $60 \%$ de poteries modelées à la main, de type " protohistorique " (mais encore attesté de nos jours), $30 \%$ de poteries "communes" tournées, probablement maghrébines et venues par la mer. Le dixième restant était composé de céramiques "à vernis noir " (dites "campaniennes») qui témoignent du commerce maritime punique à plus longue distance. Cette composition témoigne de la culture locale, restée dans l'ensemble fidèle à ses traditions et à ses pratiques, tout en étant ouverte aux échanges méditerranéens.

\section{Le contrôle punique de la côte}

7 A la fin $\mathrm{du} \mathrm{III}^{\mathrm{e}}$ siècle avant J.-C, les principales villes côtières semblent avoir été contrôlées directement par Carthage. Un extraordinaire trésor enfoui à Bejaia vers 210 avant J.-C., au cours de la seconde guerre punique (219 à 201 avant J.-C.) en témoigne (Laporte 1996; Soltani 2000). La totalité des quelques 3000 monnaies qui le composaient sont puniques, cette pureté semble montrer à cette époque la mainmise directe de Carthage sur la ville. Elle fut sans doute entraînée dans le conflit. Comme d'autres villes du Metagonium* soumises à Carthage, elle fournit probablement des soldats à l'appel d'Hannibal. 


\section{Les royaumes numides}

8 La défaite de Carthage en 201 après J.-C. ouvrit la côte aux royaumes maures et numides qui contrôlaient depuis longtemps l'intérieur. Les Kabylies étaient situées entre celui du masaessyle* Syphax* (région de Siga*) et celui du massyle* Gaia* (région de Cirta*). Elles passèrent sans doute aux mains de Syphax lorsqu'il conquit le royaume massyle, puis à celles de Massinissa lorsqu'il triompha finalement en s'appuyant sur Rome. Nous ignorons tout de l'emprise réelle de ces royaumes maures et numides sur la région, dans la mesure où la royauté portait beaucoup moins sur des territoires que sur des tribus liées au roi par un réseau d'allégeances. Nous ne savons pas situer dans le temps les vestiges qui peuvent rester de ces époques. Toutefois, une stèle libyque découverte à Kerfala*, semble bien montrer un chef massyle qualifié de GLDMȘK, chef (de tribu ?) (Chaker 1977, Salama 1992) en pleine région masaessyle. Ce petit monument appartient à une série de stèles, datables des trois derniers siècles avant notre ère (Laporte 1996), trouvées autour de la vallée du Sebaou et résumées en quelque sorte par la stèle d'Abizar*. Si la figuration de chefs existe ailleurs dans le monde libyque, les stèles de la Kabylie du Djurdjura représentent un ensemble dont l'homogénéité stylistique et culturelle n'a pas d'équivalent. Elles montrent des chefs coiffés d'une sorte de turban ( ?), la barbe en pointe. Chevauchant de petits chevaux, ils étaient armés de trois ou quatre javelines et se protégeaient d'un petit bouclier, armement de chasse (notamment contre les fauves), mais qui pouvait également les rendre redoutables à la guerre. C'est probablement de la même époque que datent les nombreuses peintures libyques découvertes sur les masses de grès éparses sur la chaîne côtière, au Nord du Djurdjura (Masso et Poyto 1969). À l'instar des multiples inscriptions peintes de la grotte d'Ifigha, révélées pour la première fois par Saïd Boulifa en 1909, elles ne montrent aucune lettre punique ou latine. 
Stèle bilingue (libyque et néo-punique) de Cissi (Cap Djinet). Musée d'Alger. Dessin J.-P. Laporte après Marcillet-Jaubert, Libyca,VIII 1960, p. 152.

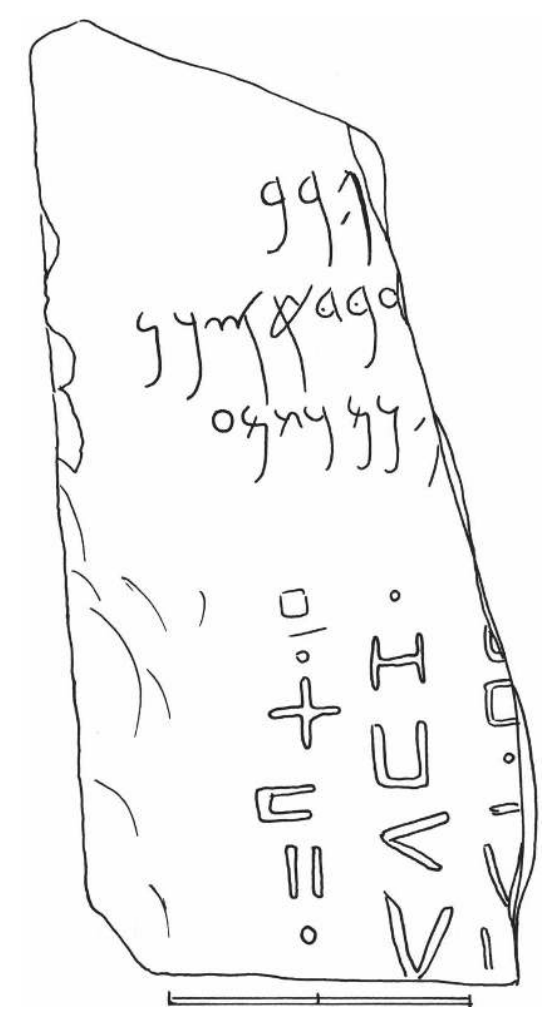

9 Avec la chute de Carthage en 146 avant J.-C, l'influence punique diminua au profit de celle de Rome, de plus en plus présente dans les échanges commerciaux, avec les villes de la côte. Pourtant celles-ci émirent des monnaies où leur nom était encore écrit en lettres puniques et non en lettres latines (ou même libyques). Le punique restait une langue de culture et d'échange. Saldae et Rusuccuru notamment émirent des monnaies de ce type (Mazard 1955, p. 160 et Laporte 1995). Une inscription néo-punique découverte fortuitement à Cap Djinet (Cissi*) concerne un certain DRK 'DNB'L, habitant de KSY (Cissi), dont le nom, Derku Adonibaal (Lipinsky 1992, p. 134), allie une partie libyque et une partie punique, ce qui montre l'interpénétration des deux cultures.

\section{La montée de l'emprise romaine}

Peu à peu l'emprise romaine grandit au point que la région fut entraînée dans les guerres civiles romaines. On vit par exemple en 44 avant J.-C. Rusuccuru (Dellys*) refuser l'entrée de Pompée le Jeune et choisir le camp des futurs vainqueurs césariens. En 33 avant J.-C, Bocchus légua son royaume au peuple romain, qui ne sut pas tout de suite qu'en faire. Après la bataille d'Actium (31 avant $\mathrm{J} .-\mathrm{C}$ ), Auguste installa des vétérans d'une même VII légion dans les trois colonies de Saldae, Tubusuctu et Rusazus (Laporte 1998). Plus à l'est, il créa également une colonie à Igilgili (Jijel). Mais en 25 avant J.-C, il finit par confier la Maurétanie césarienne à un souverain autochtone, Juba II*, fils du grand Juba*. En fait, le jeune homme avait été élevé à Rome et était proche de l'empereur. Rome contrôlait déjà le pouvoir maurétanien. Dans les villes de la côte, la culture romaine progressa peu à peu au détriment des traditions libyco-puniques. 
11 En 39 apr. J.-C, l'assassinat à Lyon du roi Ptolémée* (fils de Juba II), provoqua une insurrection conduite par l'un de ses affranchis, Aedemon. Deux trésors, trouvés l'un à Cap Djinet* (Cissi) et l'autre à Dellys* (Rusuccuru), témoignent de la participation de la région à cette aventure. L'empereur Claude fit écraser la révolte et transforma la Maurétanie en province romaine, en lui conservant apparemment à l'Est l'étendue qu'elle avait sous Juba II et Ptolémée, avec sa curieuse excroissance vers le Nord-Est englobant les Babors, géographiquement plus proches de la Numidie (Camps 1973). Au temps du danger, Rusuccuru probablement déjà fort romanisée, avait choisi le «bon camp ». Claude lui accorda le statut de municipe de droit latin, ce qui lui permettait d'entrer résolument dans le monde romain tout en conservant ses structures et lois municipales antérieures (c'est-à-dire probablement encore puniques). Pendant de nombreuses années, la Maurétanie changea peu, avec toutefois l'élévation de Sétif au rang de colonie sous Nerva (96-98).

\section{La poussée romaine sous Hadrien}

Sous Hadrien (117-138), la région connut une forte poussée romaine, tant vers le sud, avec l'installation d'une forte garnison à Auzia (Sour el Ghozlane*, ex Aumale) et la construction du camp de Rapidum* (Sour Djouab, ex Masqueray), que sur la côte avec une densification de la romanisation des villes. C'est ainsi que Choba* (Ziama, près de Mansouriah), un ancien "comptoir » punique, reçut d'Hadrien le statut de municipe. C'est vers cette époque que les stèles votives d'Iomnium et Rusippisir (Tigzirt* et Taksebt*) prirent enfin un aspect plus romain. La romanisation des villes se prolongea sous Antonin le Pieux, avec un grand effort d'équipement dont le célèbre aqueduc de Saldae (Bejaia*) est un bel exemple.

13 La pression romaine croissante sur les territoires des tribus est illustrée de manière frappante dans la région de Jijel par la dédicace en 128 d'un castellum Victoriae en limite du territoire de la tribu des Zimizes*, et probablement sur des terrains qui leur avaient été enlevés (CIL, VIII, 8369). Cette pression provoqua comme il se doit des révoltes, dont la mieux connue embrasa l'ensemble de la Maurétanie césarienne dans les années 150-160, sous Antonin le Pieux (138-161). Iomnium et probablement Rusippisir s'entourèrent d'un rempart. C'est alors qu'apparu dans les Babors une grande confédération autochtone, les Bavares* (qui ont donné leur nom aux Babors), dont la soumission fut apparemment très coûteuse, et qui devait faire parler d'elle tout au long du siècle suivant (Camps 1955). Quelque temps plus tard, c'est dans la Kabylie du Djurdjura que se forma une autre grande confédération, dont nous ne connaissons que le nom latin: les Quinquegentanei* (les Cinq Peuples), qui devait résister elle aussi à l'occupation, surtout pendant la seconde moitié du $\mathrm{III}^{\mathrm{e}}$ siècle. C'est apparemment à cette époque que Rome construisit une route fortifiée joignant les vallées de la Soummam et du Sebaou par Ksar Chebel* et Ksar Adekkar*.

14 D'un point de vue militaire, Kabylies du Djurdjura et des Babors se trouvaient dans des situations analogues : pour l'essentiel, des zones tribales montagneuses coincées entre un chapelet de villes côtières au Nord et les plaines romanisées au sud, avec deux importantes villes de garnison pour les surveiller, Auzia pour l'une, Sitifis (Sétif*) pour l'autre. 


\section{Une nouvelle poussée romaine sous Septime Sévère}

15 La romanisation de la province connut une nouvelle poussée sous Septime Sévère (198-203). Un « cens » (recensement) de la Maurétanie en 198/199, sous le procurateur C. Octavius Pudens (198-200), fut l'occasion d'un réexamen complet de l'organisation de la province. L'activité considérable notée en Césarienne sous son gouvernement se prolongea pendant celui de son successeur immédiat au poste de procurateur de Césarienne, P. Aelius Peregrinus (201-203) (Benseddik 1997). Le territoire romain fut agrandi par l'établissement au sud d'une nova praetentura ("nouvelle couverture ») au sud du Titteri et des monts du Hodna, mais aussi sans doute par une pression croissante sur les tribus du nord, au sein de territoires considérés comme romanisés depuis longtemps, à tort sans doute. On note dans la région des travaux routiers, la restauration de la tour de Daouark (près d'Azeffoun), un milliaire à Takdempt près de Dellys, une stèle élevée par un militaire à Ksar Chebel*. Du côté des villes romanisées, nombre de témoignages datent de cette époque; devant leur abondance, nous ne citerons que la dédicace du temple de Tigzirt (Iomnium), dans lequel C. Iulius Honorius, originaire de Rusuccuru, élevait une dédicace au génie de ce municipe (dont Iomnium dépendait).

16 C'est une époque pour laquelle de nombreuses inscriptions latines donnent une bonne image de la population. À l'exception des trois colonies augustéennes de Rusazus*, Salda* et Tubusuptu*, les apports romains et italiens avaient été très faibles. La population était donc pour l'essentiel d'origine africaine, avec tous les degrés de romanisation. Les villes étaient alors fortement romanisées, tant dans leur fonctionnement que dans le nom d'une partie de leurs habitants. Les municipes euxmêmes avaient vu le nombre de citoyens romains augmenter (en effet, les charges publiques permettaient d'accéder à la citoyenneté romaine), sans que les nouveaux promus oublient leurs racines, rappelées parfois par des cognomina (surnoms) libyques, voire puniques ou punicisants. La proximité des villes structurait les territoires proches, notamment dans la région d'Iomnium, où subsistent de nombreux vestiges de pressoirs à huile correspondant à de petites exploitations et non à de grands domaines (Laporte 1980). La prospérité économique allait de pair. Tubusuctu* (près d'El Kseur) exportait abondamment un produit liquide (huile ou vin?) dans des amphores d'un type particulier portant fièrement une estampille indiquant son lieu de provenance : ex provinciae Mauretaniae Caesarensi, Tubusuctu (Laporte 1980). Saldae en exportait également, avec une estampille également précise: (ex) $P$ (rovinciae) $M$ (auritaniae) C(aesariensis), Sal(dae), (de) of(ficinae) Plotior(um). Un petit port aujourd'hui ensablé, Musluvium* (Andriech), avait même à Ostie, le port de Rome, un bureau dont la mosaïque porte encore les mots Musluvitani hic: «ici (on trouve) les (négociants) de Musluvium ». Malgré toutes les destructions, cette période a laissé dans les Kabylies un grand nombre de traces archéologiques "romaines", patrimoine important qui n'est pas celui des colonisateurs étrangers, mais bien celui des habitants du pays.

\section{La population non romanisée}

Les zones laissées aux tribus restaient à l'écart. Il est significatif de noter que des secteurs entiers ne livrent que des mausolées antiques isolés (aqbu-s), sépultures «à la romaine» de chefs romanisés alors que leurs sujets l'étaient sans doute beaucoup 
moins, voire pas du tout. C'est en effet un des traits importants des deux Kabylies que de n'avoir été touchées qu'à la marge par la romanisation et d'avoir conservé des territoires tribaux très étendus. Ils ne sont guère connus que par des témoignages romains ou romanisés : inscriptions «romaines", textes historiques grecs ou latins, soigneusement recensés naguère (Desanges 1962). Une première liste donnée par le géographe Ptolémée* doit témoigner de la situation vers la fin du i ${ }^{\mathrm{er}}$ siècle après J.-C. Elle cite des peuples dont la localisation reste difficile (Desanges 1962, carte $n^{\circ} 2$ ): Toulensii, Baniouri, Moukouni, Koidamousi, Makhoures, Khitouae.

Carte des tribus attestées dans les Kabylies après le début du ॥e siècle extraite de Desanges, Catalogue des tribus, Dakar, 1962, carte 3. N.B. Les Nababes ont été repositionnés ici dans la vallée du Sebaou.

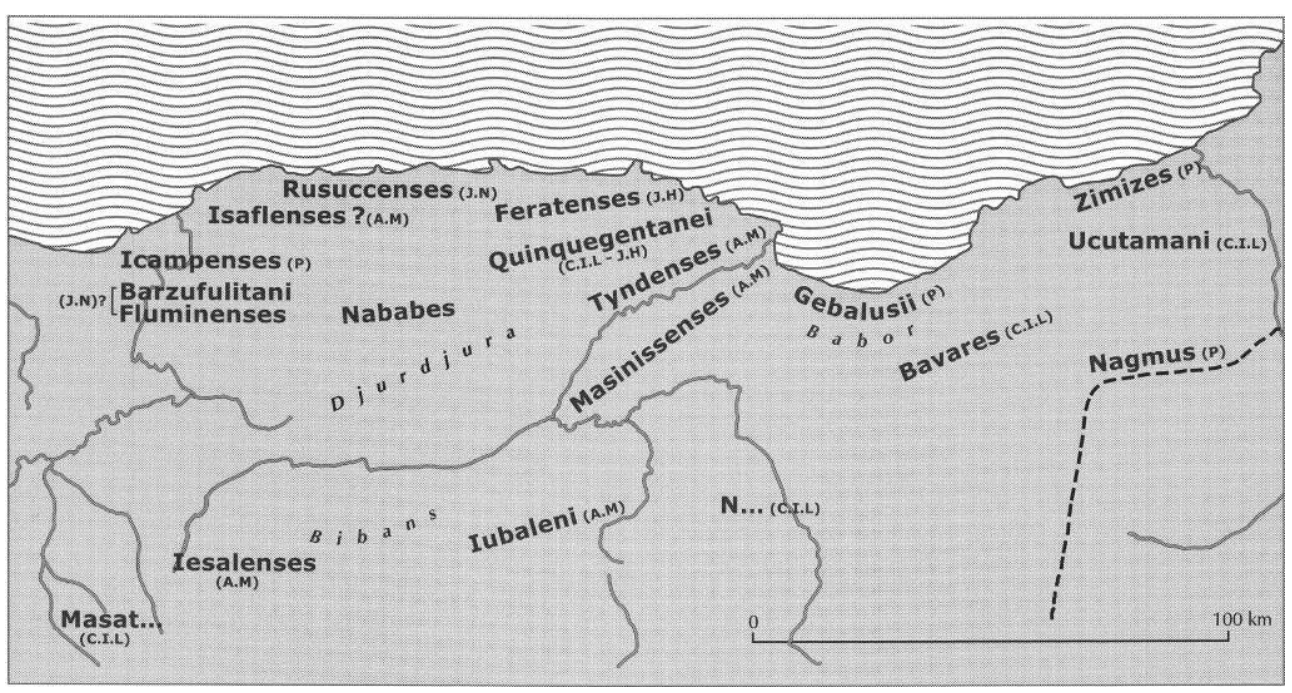

D'autres peuples, cités ou attestés plus tard, peuvent être localisés avec plus de précision (Desanges 1962, carte $\mathrm{n}^{\circ} 3$ ): Icampenses, Barzufulitani, Fluminenses, Rusuccenses, Isaflenses, Feratenses, Nababes, Tyndenses, Massinissenses, Gebalusii, Zimizes, Ucutamani. Cette liste est certainement très incomplète, et certains de ces noms peuvent désigner une partie d'un autre peuple. Deux noms désignent certainement de grandes confédérations. Il s'agit des Quinquegentanei* ( les Cinq Peuples ») de la Kabylie du Djurdjura (Galand 1970) et des Bavares de la Kabylie des Babors (Camps 1955), qui ont d'ailleurs probablement laissé leur nom à ce massif.

\section{Les chefs libyques}

Depuis le début, c'est-à-dire les alentours de notre ère, l'administration romaine avait adopté des solutions diversifiées pour gérer les tribus, en respectant autant que possible leur organisation antérieure. Elle s'était appuyée sur leurs chefs. Le plus anciennement attesté est, à Toudja *, un certain Vergi. En. Nerdocen (?), princeps d'une tribu dont le nom n'a pas été indiqué (CIL, VIII, 8984). Lorsqu'il mourut, on éleva à sa mémoire une stèle qui rappelait par sa forme les stèles libyques figurées des siècles antérieurs mais comportait déjà une dédicace latine. Il est le premier connu des notables autochtones promus par Rome, qui pouvait leur attribuer la citoyenneté romaine à titre individuel en raison de leur dévouement. Dans d'autres cas, une tribu pouvait être administrée par un officier nommé préfet (praefectus gentis), qui flanquait 
parfois le princeps laissé en place. Plus tard, certaines tribus paraissent commandées par un regulus (« roi » ou « petit roi ») dont on ne connaît pas le statut exact.

Stèle du princeps de Toudja (CIL, VIII, 8984). D'après un document Renier, Sorbonne, ms 454 (2), document 215.

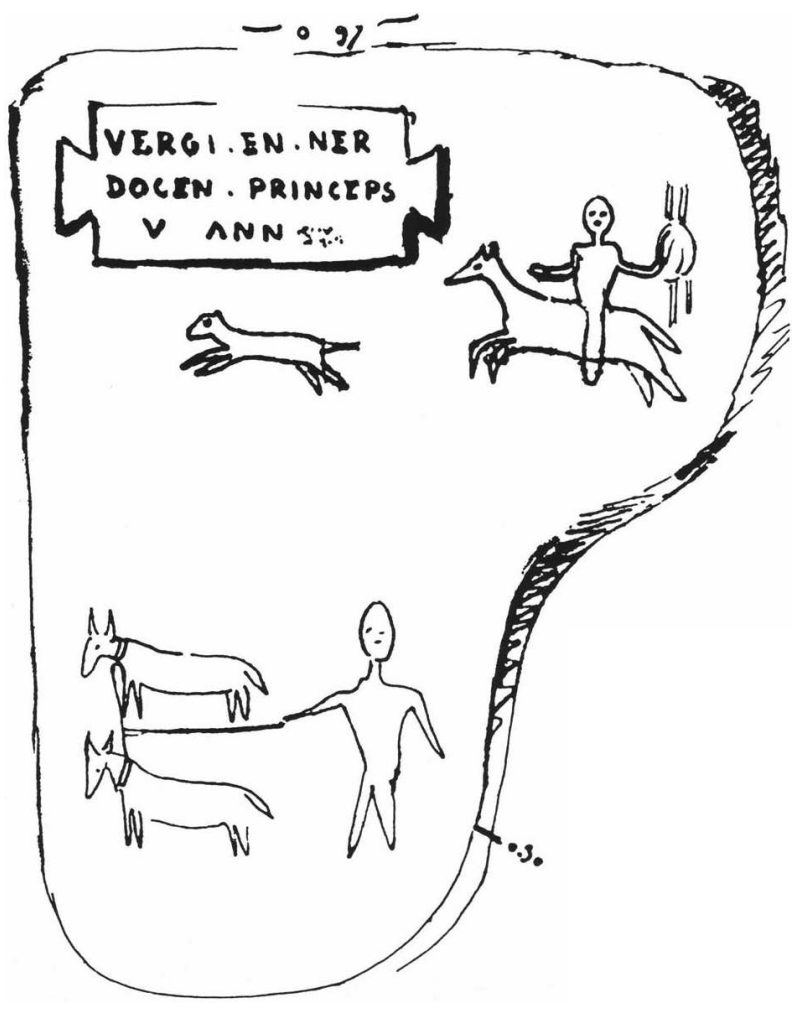

\section{Les difficultés du III ${ }^{\mathrm{e}}$ siècle}

Cette solution, propre à conserver un certain calme, et à romaniser en douceur une partie des élites autochtones, montra ses limites dès que la pression romaine sur les territoires et les finances des tribus s'accentua. Il est significatif de noter que de grandes difficultés suivirent de peu l'apogée de la puissance romaine dans la région sous Septime Sévère.

La situation ne fit que se tendre. Après le règne d'Alexandre Sévère (222-235), l'Empire romain dut faire face à des attaques extérieures (Germains, Sarmates, Perses) et se donna des empereurs soldats. En Maurétanie césarienne, c'est de l'intérieur du territoire considéré comme romain que vinrent les troubles. En 226-227, le gouverneur de Césarienne T. Licinius Hierocles dut rassembler des troupes pour vaincre une factio non autrement précisée sous les murs d'Auzia. Presque au même moment, il faisait élever des remparts autour d'une série de castella des plaines de Sétif, sans que l'on sache si les troubles ont précédé ou suivi cet effort de colonisation (Christol 1994). En 237, l'historien Hérodien présente les cohortes romaines comme aguerries par les attaques qu'elles avaient chaque jour à soutenir de la part de Maures. La région d'Auzia fut l'objet de combats. En 247, un enfant y fut tué par des barbares non nommés, à chercher probablement dans les montagnes situées au Nord, le Djurdjura. Les armes de jet et la rapidité des déplacements des guerriers libyques en faisaient des adversaires 
redoutables pour les troupes auxiliaires romaines. Ils excellaient dans les embuscades, mais faute d'organisation, étaient écrasés lorsqu'ils se risquaient à des batailles rangées en plaine. Ils se réfugiaient alors sur leurs montagnes inaccessibles.

Quelques années plus tard, éclata en Maurétanie césarienne une révolte générale dite "de 253» (qui dura en fait pendant toute la décennie 250-260), dans laquelle s'illustrèrent Bavares et Quinquegentanei (Salama 1988). La découverte de trésors à Cissi et à Iomnium montre que le soulèvement des seconds toucha aussi la région côtière. En 254, des «Barbares » étaient vaincus et dispersés près d'Auzia à Aïn Boudib. Bavares et Quinquegentanei n'hésitèrent pas à joindre leurs forces pour des expéditions en Numidie, razzias durement réprimées. En 259, ils furent battus près de Milev (Mila). Q. Gargilius Martialis, commandant de la cavalerie auxiliaire maure en garnison à Auzia, qui avait réussi à prendre et à tuer un chef célèbre, Faraxen, périt peu avant mars 260 sous les coups des Bavares. Vers cette époque, un gouverneur de Césarienne arrêta au col de Teniet el Meksen, près d'Horrea (Aïn Roua) une multitude de Bavares, conduits par trois rois (Taganim, Masmule et Falem), alors qu'ils allaient déboucher sur la plaine de Sétif (Gsell 1907).

Dédicace de Dioclétien et Maximien Hercule de Tubusuptu (Tiklat, près El Kseur) mentionnant les Quinquegentanei (1. 5 : [Quinque]ntaneorum ex Tubusuctitana [regione] C.I.L., VIII, 8836. Musée archéologique de Bejaia. Photo J.-P. Laporte.

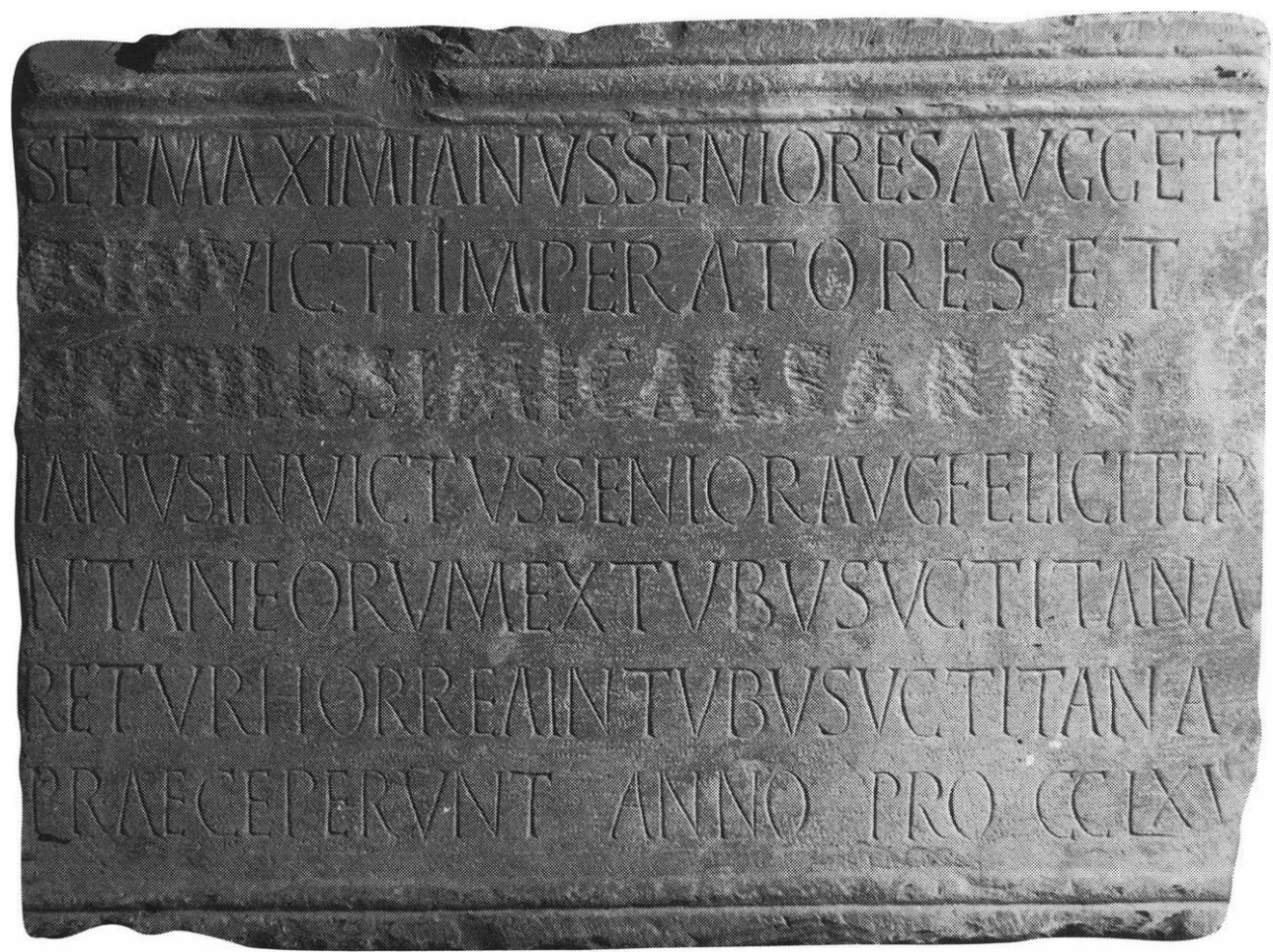

Les troubles continuèrent, probablement pas de manière continue, mais avec des périodes de paix relative interrompues par des conflits violents. Une grande révolte, marquée par plusieurs trésors, paraît avoir à nouveau secoué la région vers la fin du règne d'Aurélien (270-275) ou au début du règne de Probus (276-282) (Laporte 1980). Iomnium paraît avoir succombé peu après 270 : la ville fut détruite, probablement par les Quinquegentanei ; une épaisse couche de cendres et de décombres recouvrit les sols sévériens. Un trésor de $11 \mathrm{~kg}$ de monnaies, terminé par des divo Claudio (275-276), ne fut pas récupéré. C'est sans doute à la même époque que la petite ville de Rapidum fut prise 
et détruite par une incursion de barbares (non nommés mais dont on devine l'origine : la Kabylie du Djurdjura) et abandonnée pendant de nombreuses années (ante plurima tempora a rebellium incursione captum ac dirutum, pouvait-on écrire vers 298-300) (CIL, VIII, 20836 ; Laporte 1989, p. 239-241).

Certes, en 290, le praeses (gouverneur) de Maurétanie césarienne, T. Aurelius Litua pouvait faire élever à plusieurs endroits de sa province des stèles commémorant ses hauts faits, mais c'étaient des victoires sans lendemain. À Saldae notamment, il célébra une victoire sur les Quinquegentanei (CIL, VIII, 8924 ; Laporte 1996). Les effets ne s'en firent pas sentir bien longtemps. À peine sept ans plus tard, en 297, l'empereur Maximien Hercule dut venir les réduire en personne (Rebuffat 1992). Loin d'une promenade militaire, la campagne semble avoir été dure. Les Quinquegentanei furent vaincus et en partie déportés. Tubusuctu fut certainement l'une des ses principales bases dans cette guerre. Lors de son passage, l'empereur y décida d'importantes constructions en rapport avec la surveillance des Quinquegentanei. Loin d'avoir abandonné des territoires comme on l'avait cru, l'Empire en avait reconquis. Il avait rétabli l'ordre romain pour de longues années.

Il prit acte des réalités géographiques et militaires en scindant en deux la Césarienne. La région orientale, avec Sétif pour capitale, devint la Maurétanie sitifienne en 303 (Laporte 1996). À l'Est, elle englobait la basse vallée de la Soummam, avec Saldae et Tubusuptu. La nouvelle limite, presque nord-sud, passait quelque part dans la forêt de Yakouren, entre Ksar Chebel et Ksar Adekkar (Laporte 1998).

\section{Le début du IVe siècle}

La guerre fut suivie d'une profonde réorganisation de la province. Les inscriptions devenues rares depuis deux décennies, redevinrent plus nombreuses, notamment à Saldae. Auzia devient le siège d'un limes* citépar la Notitia dignitatum et confié à un praepositus limitis Audiensis, dont le premier est attesté par une inscription datée de 301 (CIL, VIII, 9025). Il en fut sans doute de même à Tubusuptu. La reprise en main militaire se doubla d'un renouveau de la société civile, bien visible dans les inscriptions édilitaires d'Auzia et de Saldae.

Dans le même temps, le christianisme commençait à se développer. Le brassage de population lié à l'activité portuaire avait dû assez tôt amener des chrétiens sur la côte. Sainte Marcienne, martyrisée à Césarée en 299, était originaire de Rusuccuru. C'est l'une des plus anciennes mentions de chrétiens en Maurétanie Césarienne. La Paix de l'Eglise (311) permit sans doute à la petite communauté de s'épanouir au cours du IV siècle, en investissant d'abord les villes, puis en convertissant les notables tribaux. Dellys notamment a livré un important sarcophage chrétien représentant les miracles du Christ qui doit avoir été importé de Rome vers 350.

\section{La seconde moitié du IV ${ }^{\mathrm{e}}$ siècle}

Pour tenter de garder les tribus sous contrôle, Rome avait continué à s'appuyer sur des notables autochtones, les difficultés extérieures de l'Empire paraissent leur avoir laissé une autonomie de plus en plus grande, constituant ainsi peu à peu ce qui commença à ressembler à une féodalité. 
$29 \mathrm{Au}$ milieu $\mathrm{du} \mathrm{IV}^{\mathrm{e}}$ siècle, régnait ainsi sur la région, pour le compte des autorités romaines, un certain Nubel*, regulus per nationes Mauricas potentissimus (Ammien Marcellin, XXIX, 2). À sa mort, son fils, sans doute âné et légitime, Sammac* lui succéda, et se fit construire à M’lakou, dans la vallée de la Soummam, une résidence construite "à la manière d'une ville ». La superbe dédicace découverte en 1901 (voir EвXIX, 1997, p. 2 848), aujourd'hui au Musée d'Alger, permet de bien cerner la nature de son pouvoir qui évoque quelque peu celui des "grands caïds » utilisés plus tard par d'autres colonisateurs. En leur conférant une autorité sur d'autres tribus que les leurs, on leur donnait ainsi une arme qui pouvait le cas échéant se retourner contre l'occupant. C'est ce qui se passa pour Firmus, un autre fils de Nubel. Sans doute issu d'une concubine, il n'avait pas hérité du pouvoir de son père et se l'appropria en assassinant Sammac avant 370. Condamné à mort par le comte d'Afrique Romanus, il ne put se défendre devant l'empereur et fut acculé à la rébellion. En s'appuyant sur son pouvoir sur les tribus, il les souleva (Laporte 2004). Également grand personnage romain, il réussit à s'attirer des sympathies dans les cités romaines (par ailleurs écrasées par la lourdeur de la fiscalité impériale). Les catholiques ayant été précédemment favorisés par l'autorité impériale contre laquelle il se révoltait, les donatistes se rallièrent à lui. Un évêque donatiste, le plus ancien attesté en Kabylie, ouvrit aux troupes de Firmus les portes de Rusuccuru (Laporte 1994, p. 435-437). Pendant plusieurs années, la Maurétanie césarienne fut considérée comme perdue pour l'Empire. En 373, l'empereur Valentinien envoya le général Théodose (père du futur empereur de ce nom) réduire la rébellion. Il débarqua à Igilgili, rassembla ses troupes dans la région de Sétif et passa à l'action en commençant par Tubusuctu. Remontant la vallée de la Soummam, il dévasta le praesidium de Sammac, puis soumit diverses tribus, notamment Tyndenses et Massinissensiz avant de se diriger vers Icosium (Alger), Tipasa et Cherchel en passant par la Kabylie du Djurdjura. Il lui fallut plus d'un an pour soumettre la province révoltée. Les effets de la guerre furent dévastateurs tant pour les villes que pour les tribus. La province tout entière fut très affaiblie, au point d'obtenir plus tard des dégrèvements fiscaux.

\section{Le siècle vandale}

Ensuite, la vie reprit lentement. Au milieu de ces difficultés, la grande basilique de Tigzirt, peut-être antérieure à la révolte de Firmus, témoigne de la vigueur de l'implantation chrétienne sur la côte (Laporte 1994). Une liste des évêques présents au concile de Carthage en 411 montre des évêques dans les principales villes de la région. Compte tenu des difficultés de l'Empire en Europe et en Orient, la région paraissait encore prospère au début du v siècle. Pour peu de temps.

Les Vandales, passés de Germanie en Gaule en 406, et stationnés depuis en Espagne, débarquèrent en Afrique en 429. Ils longèrent la mer vers l'Est, en semant la désolation sur leur passage. Ils passèrent sans aucun doute par les Kabylies, bien que nous n'en ayons pas de témoignage. En 430, saint Augustin mourut dans Hippone assiégée. Carthage même succomba en 439 et Genséric y transporta sa capitale. C'est probablement à cette époque qu'il fit détruire les remparts des villes romaines de Maurétanie césarienne pour les mettre à la merci des tribus et être ainsi plus tranquille sur ses arrières. 
32 L'Empire fut obligé de traiter et récupéra en 442 la Maurétanie. La province était ruinée. Les affaires religieuses étaient également en difficulté, et le pape saint Léon avait dû dès avant 445 prendre la direction des églises maurétaniennes. En 454, la Maurétanie césarienne sortit de l'Empire, les villes côtières jusqu'à Caesarea (Cherchel) restant aux Vandales, tandis que dans l'intérieur du pays, laissé enfin à lui-même, se créaient des royaumes berbères chrétiens (illustrés par les Djedars* de la région de Tiaret). L'ancienne Maurétanie sitifienne, dont faisaient partie les Babors, fut prise en main par les Vandales, qui y favorisèrent l'arianisme. En 484, nombre d'évêques catholiques de la région furent bannis ou emprisonnés sur l'ordre du roi Hunéric. Ce fut le cas des évêques d'Igilgili, Choba, Saldae et Tubusuctu notamment, qui furent effectivement exilés. Les deux derniers cités furent les plus occidentaux à subir cette peine. Ceci paraît montrer que la vallée de la Soummam constituait la limite occidentale du royaume vandale (Modéran 1999, carte p.262), comme elle avait constitué jadis celle de la Maurétanie sitifienne.

Plus à l'Ouest, la majeure partie de la province échappait à l'autorité vandale. La situation des établissements côtiers paraît avoir été précaire. À Iomnium, la grande basilique avait été détruite par un incendie (consécutif peut-être à un tremblement de terre) sans doute dans la première moitié du v siècle. Alors qu'il aurait été possible de la restaurer, on se borna à installer une église plus petite au milieu de la nef en ruine (Laporte 1994). L'évêque avait abandonné Iomnium au profit de Rusippisir où il résidait en 484 .

Au milieu de cette décadence de la vie à la romaine, naissait autre chose. Nous avons vu que, plus à l'ouest, des royaumes indépendants se constituaient à l'intérieur du pays. Il se passait aussi quelque chose, sans que nous sachions exactement quoi, dans les Kabylies. Dans la région du bas Isser, près de Blad Guitoun, on élevait, sans doute au v siècle, un grand mausolée octogonal (parfois encore faussement attribué à Firmus contre toute vraisemblance) pour abriter les restes d'un chef chrétien (Gsell 1898). Orné d'une abondante décoration souvent géométrique et en partie chrétienne, il reprenait des dispositions de culte funéraire venues des plus lointains horizons libyques, traditions restées vivantes à côté de la romanité qui les avait en apparence supplantées pendant plusieurs siècles.

Plus haut dans les montagnes du Djurdura, des chapiteaux tardifs, certes mal datés, semblent montrer une pénétration du christianisme plus étendue qu'on ne l'a cru, avec une expansion continue même après la disparition de l'autorité romaine (Benseddik 1989, p. 737-738 et pl. I-II).

\section{Le siècle byzantin}

Le royaume vandale s'effondra en quelques mois à partir du débarquement byzantin à Caput Vada, Ras Kaboudia (Tunisie) en 533. L'Empire eut l'ambition de recouvrer l'ensemble de l'Afrique. En 534, Justinien reconstitua sur le papier deux provinces de Maurétanie. Mais en Maurétanie césarienne, les Byzantins furent tenus en échec par les royaumes berbères indépendants qui s'étaient constitués à partir des années 480 . Ils durent se contenter d'aménager sommairement des retranchements de surface réduite dans les escales qui permettaient d'accéder à la capitale, Caesarea (Cherchel). Les villes de la côte kabyle reçurent de petites garnisons byzantines. Restés longtemps inoccupés par la suite, les sites de Tigzirt (Iomnium) et Ziama (Choba) ont livré les vestiges de ces 
installations sommaires. Les Byzantins paraissent avoir trouvé Iomnium en ruines, réduite à quelques masures. L'enceinte $d u$ second siècle, probablement ruinée en partie, était beaucoup trop grande. Pour mieux assurer la défense de l'escale, on réduisit le périmètre, en abandonnant les principaux monuments de la ville antérieure. Seule la pointe de Tigzirt fut réoccupée; un nouveau rempart construit en remplois protégea une surface très réduite : 1,5 ha (contre 8 ha environ pour la ville antérieure), ce qui montre la modestie des moyens effectivement déployés. Choba fut aménagée de même (Laporte 1988, 1994).

Tigzirt, antique lomnium. Plan (retouché) d'après Gsell, Atlas, 1911, f. VI, n 34.

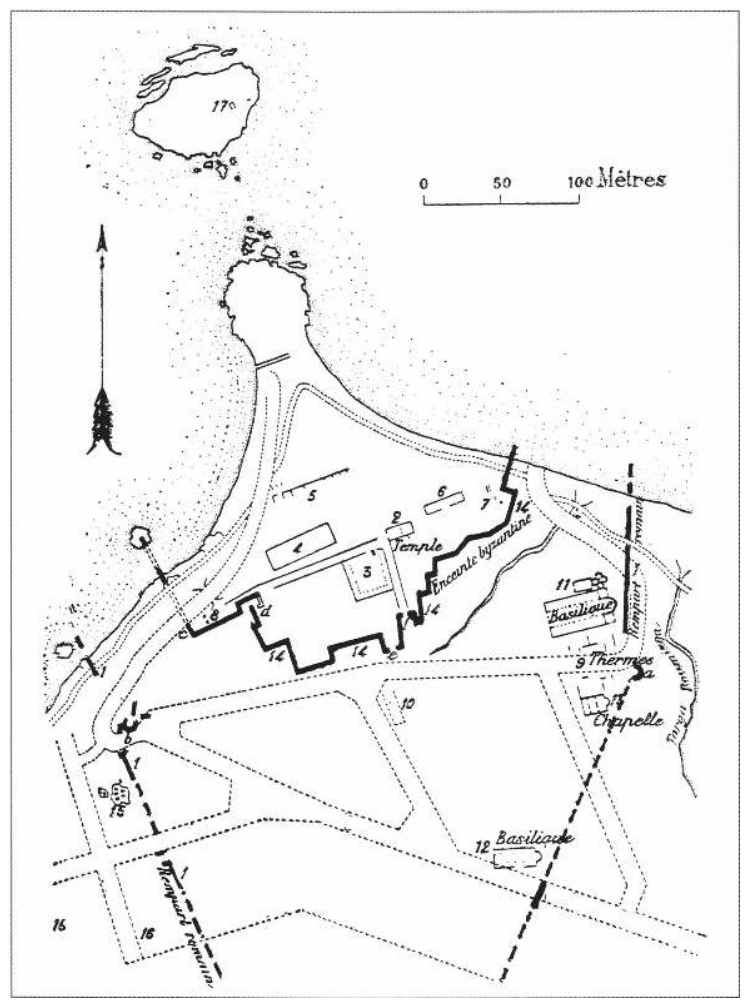

Les rues en pointillé sont celles du village moderne. La grande enceinte date du ॥e siècle après J.-C. Le rempart byzantin isole la pointe. À comparer au plan de Choba, ci-dessus, fasc. XIII, p. 1934. 


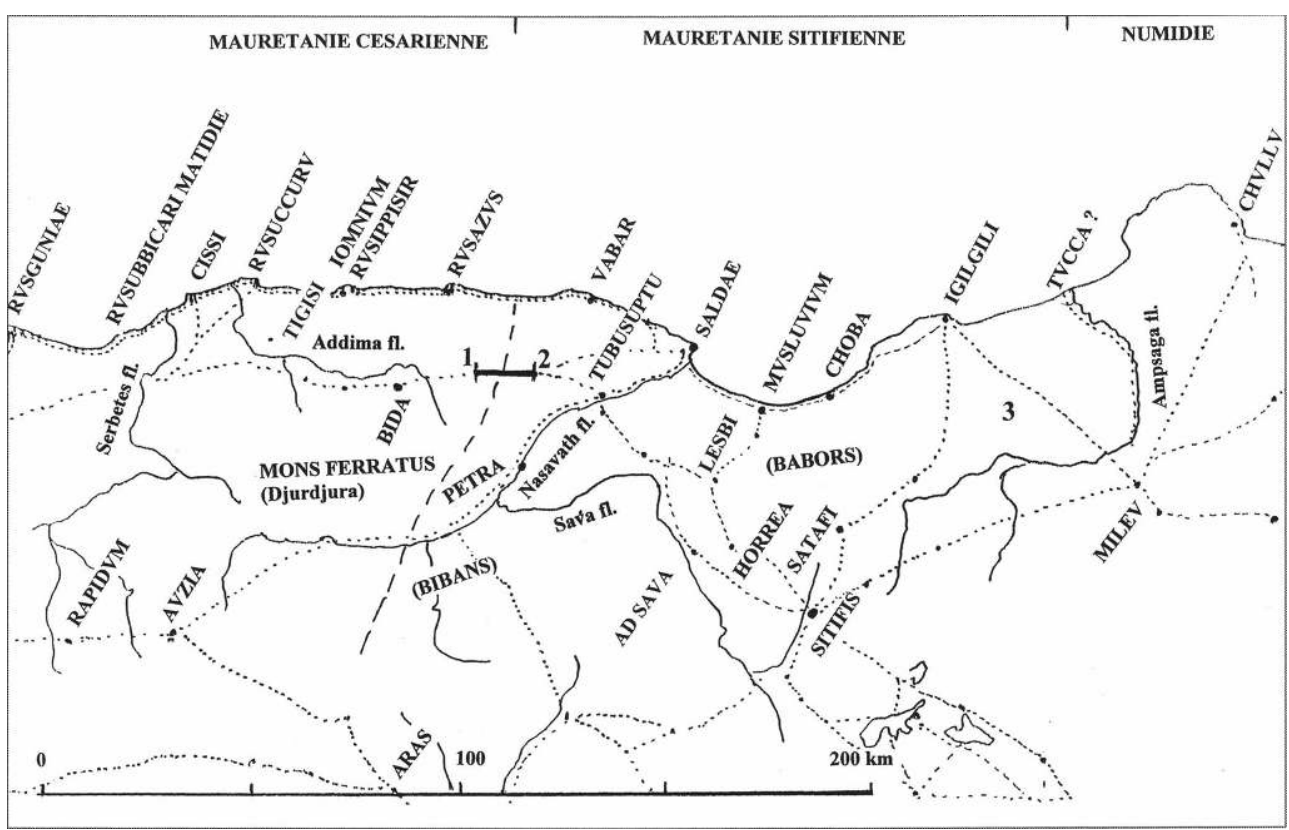

1. Ksar Chebel - 2. Ksar Adekar - 3. Col de Fdoulès. $\mid=$ = = = = | route fortifiée entre 1 et 2 („I siècle). capitale officielle et résidence d'un dux (chef militaire). Ils en furent peut-être chassés dès la fin $\mathrm{du} \mathrm{vi}^{\mathrm{e}}$ siècle. Les escales côtières qui n'avaient plus d'objet furent sans doute évacuées. Nous n'avons aucun indice sur ce qui se passa ensuite

À une date inconnue, mais probablement à l'époque byzantine, un rex gentis Ucutaman(orum) fit graver une dédicace sur un rocher du col de Fdoulès, dans les Babors $($ CIL, VIII, $8379=20216)$. Il régnait sur les Ucutumani déjà attestés au $\mathrm{I}^{\mathrm{er}}$ siècle (Koidamousoi de Ptolémée), dans lesquels il faut reconnaître les Kutama* qui devaient construire l'empire fatimide. On trouve ici l'une de ces continuités à travers les siècles que l'on commence à mettre en valeur un peu partout dans l'histoire du Maghreb. La puissance byzantine décrut peu à peu après la sécession de l'Africa en 647 , puis les premières incursions arabes dans le Sud, jusqu'au Hodna d'abord, puis plus à l'Ouest. Toute la région fut laissée à elle-même, sans que nous sachions ce qui s'y passa pendant plusieurs siècles.

\section{La fin du Christianisme autochtone}

L'islam et la puissance musulmane ne s'installèrent que très progressivement, relativement rapidement au sud, dans les régions de Sétif et du Hodna, et sans doute tardivement sur la côte et dans les montagnes kabyles. La rareté des sources ne permet pas d'en dire plus. Comme on le sait maintenant pour nombre d'autres régions au Maghreb, certaines communautés chrétiennes se sont maintenues fort longtemps (au moins jusqu'au $\mathrm{XI}^{\mathrm{e}}$ siècle dans la ville sainte de Kairouan*!). La région en donne un exemple frappant. Lorsque la Kalaa des Beni Hammad fut menacée par les invasions arabes, En-Nacer fonda une autre capitale (sous le nom d'En-Naciria) dans l'ancienne Saldae (Bejaia). Des chrétiens y vivaient encore, coupés du siège apostolique. En 1076, l'émir de Bougie El Moufok, envoya au pape un bougiote nommé Servandus (un nom 
latin) qui avait été élu évêque par les chrétiens de sa ville, pour lui demander de le consacrer, modèle de tolérance religieuse qui pourrait encore servir aux hommes de notre temps. Grégoire VII lui répondit favorablement. Il écrivit parallèlement une lettre adressée "clero et populo Buzee in Mauritania Sitifense " c'est-à-dire "au clergé et au peuple de Bougie » (Lancel 2003, p. 112-115). C'est à ce jour la dernière mention connue de chrétiens autochtones, le dernier signe de l'Antiquité romaine, puis chrétienne, dans la région.

\section{Correspondance entre les noms antiques et modernes}

\begin{tabular}{|c|c|}
\hline Nom antique & Nom actuel \\
\hline Rusguniae & Cap Matifou \\
\hline Rusbbicari & Port aux Poules \\
\hline Modunga & Site non retrouvé \\
\hline Serbétes flumen & Oued Isser \\
\hline Cissi $\quad \ldots \ldots$ & Cap Diinet \\
\hline Adima flumen & Embouchure du Sebaou \\
\hline Rusuccuru & Dellys \\
\hline Iomnium & Tigzirt \\
\hline Rusippisir & Taksebt \\
\hline Rusazus & Azeffoun (ex Port-Gueydon) \\
\hline Vabar & Site de l'Oued Daas \\
\hline Saldae & Bougie - - \\
\hline Nasabath flumen & Embouchure de la Soummam \\
\hline Musluvium & Andriech \\
\hline Choba & Ziama (près Mansouriah) \\
\hline Igilgili & Jijel (Djidjelli) \\
\hline Ampsaga & Oued el Kebir \\
\hline \multicolumn{2}{|c|}{ Route moyenne du Djurdjura } \\
\hline Tubusuptu & Tiklat (près El Kseur) \\
\hline Bida & Djemaa Saharidj \\
\hline Tigisi & Taourga (ex-Horace Vernet) \\
\hline Castellum Tulei & - \\
\hline$?$ & Dra-Zeg-et-Ter \\
\hline$?$ & Blad Guitoun (ex-Félix Faure) \\
\hline \multicolumn{2}{|l|}{ Intérieur } \\
\hline Rapidum & Sour-Diouab (ex Masqueray) \\
\hline Auzia & Sour-el-Ghozlane (ex Aumale) \\
\hline Petra & M'lakou \\
\hline Sava flumen & Oued Bou Sellam \\
\hline Ad Sava munic. & Hammam Guergour \\
\hline Sifitis & Sétif \\
\hline Satafi & Ain-el-Kebira (ex-Périgotville) \\
\hline Milev & Mila \\
\hline
\end{tabular}

\section{BIBLIOGRAPHIE}

BENSEDDIK N., « Nouvelles contributions à l'Atlas archéologique de l'Algérie », Africa Romana, VII, 1989 [1990], p. 737-751.

BENSEDDIK N., «P. Aelius Peregrinus Rogatus et la praetentura de Maurétanie Césarienne », Frontières et limites géographiques de l'Afrique du Nord antique, Centre de Recherche sur l'Antiquité Tardive et le Haut Moyen Âge, Paris, mai 1997, p. 89-107.

CAMPS G., « Les Bavares, peuples de Maurétanie césarienne », Revue africaine, t. 99, 1955,

p. 241-288. 
CAMPS G., « Une frontière inexpliquée, la limite de la Berbérie orientale de la protohistoire au Moyen Âge ", in : Maghreb et Sahara : études géographiques offertes à Jean Despois, Paris, Société de Géographie, 1973, p. 59-67.

CAMPS G., CHAKER S., LAPORTE J.-P, « Deux nouvelles stèles kabyles au cavalier », BCTH, n.s., Afrique du Nord, fasc. 25, 1996-1998 (1999), p. 19-32. CHAKER S., « Une inscription libyque au Musée des Antiquités d'Alger », Libyca, A/P/E, 25, 1977, p. 193-202.

CHRISTOL M., « Les troubles en Maurétanie césarienne sous le gouvernement de T. Licinius Hiéroclès ", Mélanges Le Glay = Latomus, 226, 1994, p. 255-256.

DESANGES J., Catalogue des tribus africaines de l'Antiquité classique à l'ouest du Nil, Dakar, 1962.

GALAND L., «Les Quinquegentanei », BAA, IV, 1970, p. 297-299.

GSELL S., « Mausolée de Blad Guitoun », CRAI, 1898, p. 481-492.

GsELl S., (Inscription de Teniet-el-Meksen), BCTH, 1907, p. CCXXVIII-CCXXIX.

GSELL S., Atlas archéologique de l'Algérie, 1911, feuilles VI et VII.

LANCEL S., Pax et concordia, Chrétiens des premiers siècles en Algérie, II ${ }^{e}-\mathrm{VII}{ }^{e}$ siècles, éd. Marsa, Alger, 2003.

LAPORTe J.-P., « Cap Djinet : Une dédicace des Cissiani à Sévère Alexandre », BCTH, n.s., B, 9, 1973, p. 25-37.

LAPORTE J.-P, « L'huile de Maurétanie Césarienne et les amphores de Tubusuctu », BCTH, n.s., B, 12-14, 1976-1978 (1980), p. 131-157.

LAPORTE J.-P, « Trésors de Maurétanie Césarienne enfouis sous Aurélien », Bulletin de la Société française de Numismatique, mai 1980, p. 695-697.

LAPORTE J.-P, « Rapidum : le camp de la Cohorte des Sardes », BSNAF, 1983, p. 253-267.

LAPORTE J.-P, « Fermes, huileries et pressoirs de Grande Kabylie », BCTH, n. s., 19, 1983 (1985), p. $127-146$.

LAPORTE J.-P, Rapidum, le camp de la cohorte des Sardes en Maurétanie césarienne, Sassari, 1989, 305 p.

LAPORTE J.-P, « Ziama-Mansouriah, antique Choba municipium », Actes du Premier Congrès international sur l'histoire de Sétif, 1988 (1994), p. 45-82.

LAPORTE J.-P, « Stèles libyques figurées de Grande Kabylie », Africa romana, IX, 1991 (1992), p. 389-423.

LAPORTE J.-P, Notice « Choba » (Ziama-Mansouriah), Encyclopédie berbère, t. XIII, 1994, p. 1933-1935.

LAPORTE J.-P, Notice « Cissi » (Cap-Djinet), Encyclopédie berbère, t. XIII, 1994, p. 1977-1980.

LAPORTE J.-P, Notice « Dellys » (antique Rusuccuru, médiévale Tedelles) », Encyclopédie berbère, XV, 1995, p. 2255-2261.

LAPORTE J.-P, « Le statut municipal de Rusuccuru », Africa Romana, t. X, 1994, p. 419-438.

LAPORTE J.-P, « La grande basilique de Tigzirt », BSNAF, 1994, p. 249 - 270. LAPORTE J.-P, « Notes sur l'aqueduc de Saldae (Bougie) », Africa romana, t. XI, 1996, p. 711-762.

LAPORTE J.-P, « Notes sur Auzia (Sour-el-Ghozlane, ex-Aumale), en Maurétanie césarienne », BSNAF, 1996 (1998), p. 300-317. 
LAPORTE J.-P, « Une inscription de Saldae (CIL, VIII, 8924) et la date de séparation des Maurétanies césarienne et sitifienne », Africa romana, t. 12, 1996 (1998), p. 1111-1121.

LAPORTE J.-P, « La limite entre les Maurétanies césarienne et sitifienne », Frontières et limites géographiques de l'Afrique du Nord antique, Hommage à P. Salama, 1998, p. 213-219.

LAPORTE J.-P, « La Legio VII et la déduction des colonies augustéennes de Césarienne », Les légions de Rome sous le Haut Empire, Actes du congrès de Lyon, septembre 1998 (2000), p. 555-579.

LAPORTE J.-P, « Exploration archéologique de la Kabylie du Djurdjura », Africa romana, t. 13, 1, 1998 (2000), p. 687-723.

LAPORTE J.-P, « Saldae : un trésor de monnaies puniques », BSNAF, 1998, p. 211-225.

LAPORTE J.-P, « Inscriptions antiques de Tiklat, antique Tubusuctu », Ubique amici (Mélanges Lassère), 2001, p. 249-283.

LAPORTE J.-P, « Le Metagonium et les échelles puniques », in L'Algérie au temps des royaumes numides, catalogue de l'exposition de Rouen, 2003, p. 50-52.

LAPORTE J.-P, « Les armées romaines et la révolte de Firmus (370-373) », L'Armée romaine de Dioclétien à Valentinien Ier (Congrès de Lyon, 2002), 2004, p. 269-288.

LIPINSKY E., S.v. « Djinet (cap) », Dictionnaire de la civilisation phénicienne et punique, 1992, p. 134.

MARTIN Père J., Bida Municipium en Maurétanie césarienne, F.D.B., 1969, 141 p.

MAZARD J., Corpus Nummorum Numidiae Mauretaniaeque, 1955, 164 p.

MODÉRAN Y., «Les frontières mouvantes du royaume vandale », in Frontières et limites géographiques de l'Afrique du Nord, Hommage à P. Salama, Paris, Sorbonne, 1999, p. 241-264.

MUSSO J.-C. et PoYTo Père G., Corpus des peintures rupestres de Grande Kabylie, Mémoires du CRAPE, AMG, 1969, 124 p., IX pl.

REBUfFAT R., « Maximien en Afrique », Klio, 74, 1992, p. 843-861. Salama P., « A propos d'une inscription libyque du Musée des Antiquités d'Alger ", À la croisée des études libyco-berbères. Mélanges offerts à Paulette Galand-Pernet et L. Galand, Paris, 1992, p. 127-140.

SALAMA P., «Vues nouvelles sur l'insurrection maurétanienne dite de 253. Le dossier numismatique ", L'Armée et les affaires militaires, Colloque international d'histoire et d'archéologie de l'Afrique du Nord, Strasbourg, 1988, Paris, (1991), p. 457-470.

SALAMA P. et LAPORTE J.-P, « Les chefs libyques sur les stèles figurées », L'Algérie au temps des royaumes numides, catalogue de l'exposition de Rouen, 2003, p. 33-35. Soltani A., « À propos du trésor monétaire punique de Bougie (Algérie) », Africa romana, 13, 1998, p. 1 779-1 782.

VIGNERAL C. de, Ruines romaines de l'Algérie. Kabylie du Djudjura, 1868, ... p., XVI pl.

\section{INDEX}

Mots-clés : Antiquité, Byzantins, Chefferie, Christianisme, Histoire, Kabylie, Ketama, Libyque, Protohistoire, Punique, Résistance, Rome, Royaumes numides, Tribu, Vandales 